\title{
ON $k$-SPACES AND FUNCTION SPACES
}

R. W. BAGLEY AND J. S. YANG

Let $F$ be a family of continuous functions from a topological space $X$ to a topological space $Y$. Denote by $\mathcal{e}$ the compact-open topology on $F$. In this paper we consider the problem of when the product of two topological spaces is a $k$-space and the related question: When does compactness of $(F, \mathfrak{e})$ or, more general, local compactness imply that $\mathfrak{C}$ is jointly continuous? It is shown that the product of a locally compact Hausdorff space with a Hausdorff $k$-space is a $k$-space. This is used to prove that $\mathfrak{C}$ is jointly continuous when $(F, \mathcal{C})$ is locally compact and $X$ is a Hausdorff $k$-space. These results are combined with a theorem of $\mathrm{R}$. Arens [1] to construct an example of two $k$ spaces whose product is not a $k$-space. We also prove a generalization of the Ascoli Theorem 7.21 [2, Theorem 21, Chapter 7]. In a remark following this theorem Kelley points out that it can be extended to $k$-spaces by weakening the condition on even continuity. We show that the theorem holds for Hausdorff $k$-spaces without alteration, Theorem 4. The same remark holds for [2, Theorem 7.17].

Theorem 1. If $X$ is a locally compact Hausdorff space and $Y$ is a Hausdorff $k$-space, then $X \times Y$ is a $k$-space.

Proof. Let $C$ be a subset of $X \times Y$ which intersects every compact set in a closed set. Let $(x, y) \in \bar{C}, V$ be a compact neighborhood of $x$ and $U$ any compact neighborhood of $x$ contained in $V$. Define $T=\pi_{1}(C \cap(V \times\{y\}))$ and $S=\pi_{2}(C \cap(U \times Y))$ where $\pi_{1}, \pi_{2}$ are the projections into $X, Y$ respectively. If $A$ is any compact subset of $Y$, then $S \cap A=\pi_{2}(C \cap(U \times A))$. Thus, $S$ is closed since $Y$ is a $k$-space and Hausdorff. If $W$ is a neighborhood of $y$, then $C \cap(U \times W) \neq \varnothing$ and $S \cap W=\pi_{2}(C \cap(U \times W)) \neq \varnothing$. Thus, it follows that $y \in S$ and $U \cap T \neq \varnothing$. Since $T$ is closed and $U$ was an arbitrary compact neighborhood of $x$ contained in $V, x \in T$ and hence $(x, y) \in C$. The proof is complete.

Lemma. Let $X$ and $Y$ be Hausdorff spaces, $F \subset C(X, Y)$ and let $\tau$ be a topology on $F$ which contains $\mathcal{C}$ and such that $(F, \tau) \times X$ is a $k$-space. Then $\tau$ is jointly continuous for $F$.

Proof. Let $C$ be a closed subset of $Y$ and $K$ a compact subset of 1965.

Received by the editors October 24, 1964 and, in revised form, September 21, 
$(F, \tau) \times X$. Let $M=K \cap P^{-1}(C)$ and $(f, x) \notin M$, where $P$ is the evaluation mapping of $F \times X$ into $Y$. If $(f, x) \notin K$, then obviously $(f, x) \notin \bar{M}$. Suppose $(f, x) \in K$ and $(f, x) \notin P^{-1}(C)$. Let $U=Y-C$ and $K_{X}$ be the projection of $K$ into $X$. There is a compact neighborhood $N$ of $x$ relative to $K_{X}$ such that $f(N) \subset U$ and $P([N, U] \times N) \subset U$, where $[N, U]=\{g \in F \mid g(N) \subset U\} \in \mathrm{C} \subset \tau$. Thus, $([N, U] \times N) \cap P^{-1}(C)$ $=\varnothing$. It follows that $(f, x)$ is not in the closure, relative to $(F, \tau)$ $\times K_{X}$, of $M$. But, since $M \subset K \subset F \times K_{X}$, we have $(f, x) \notin \bar{M}$. Since $F \times X$ is a $k$-space, $P^{-1}(C)$ is closed and the proof is complete.

The product of two $k$-spaces need not be a $k$-space. As a matter of fact the example below shows that, even if one of the spaces is metric, the product need not be a $k$-space. We have not been able to settle the question whether the product of two hereditary $k$-spaces is a $k$ space.

Example. Let $X$ be the dual space of an infinite dimensional Fréchet space with the compact-open topology. As Warner [4, p. 267] points out, $X$ is a hemicompact $k$-space which is not locally compact. Now $F=C(X,[0,1])$ with the compact-open topology is metrizable, [4, Theorem 2]. Suppose $X \times F$ is a $k$-space. Then, by the Lemma the compact-open topology is jointly continuous. Since $X$ is completely regular, it follows from [1, Theorem 3$]$ that $X$ is locally compact which is a contradiction, and consequently the product $X \times F$ is not a $k$-space. It follows from [3, Proposition 4] that $X \times F$ is paracompact.

The following is a generalization of (b) $[1$, p. 486]. (Cf. [4, Theorems 13 and 17].)

REMARK. If $X$ is completely regular and $X \times C(X,[0,1])$ is a $k$ space, where $C(X,[0,1])$ has the compact-open topology, then $X$ is locally compact.

Proof. The proof is immediate using [1, Theorem 3] and the Lemma.

From Theorem 1 and the Lemma we have,

Theorem 2. If $(F, \mathfrak{e})$ is locally compact, $X$ a Hausdorff $k$-space and $Y$ Hausdorff, then $\mathcal{C}$ is jointly continuous for $F$.

Using Theorem 2, we now have generalizations of [2, Theorem 7.17 and Theorem 7.21]. The proofs are the same as Kelley's by virtue of Theorem 2.

THEOREM 3. Let $X$ be a Hausdorff $k$-space and $Y$ a Hausdorff uniform space. Let $F \subset C(X, Y)$. Then $(F, \mathfrak{e})$ is compact if and only if

(a) $(F$, e) is closed. 
(b) $F(x)$ has compact closure for each $x \in X$.

(c) $F$ is equicontinuous.

TheORem 4. Let $X$ be a Hausdorff $k$-space and $Y$ a regular Hausdorff space. Let $F \subset C(X, Y)$. Then $(F, \mathfrak{e})$ is compact if and only if

(a) $(F, \mathfrak{e})$ is closed.

(b) $F(x)$ has compact closure for each $x \in X$.

(c) $F$ is evenly continuous.

Added in proof. T. S. Wu has referred us to a paper of D. E. Cohen (Spaces with weak topology, Quart. J. Math. Oxford Ser. 5 (1954), $77-80)$ in which a theorem of J. H. C. Whitehead was used to obtain Theorem 1. The proof here is direct and simpler.

\section{REFERENCES}

1. R. Arens, A topology for spaces of transformations, Ann. of Math. (2) 47 (1946), 480-495.

2. J. Kelley, General topology, Van Nostrand, New York, 1955.

3. E. Michael, A note on paracompact spaces, Proc. Amer. Math. Soc. 4 (1953), 831-838.

4. Seth Warner, The topology of compact convergence on continuous function spaces, Duke Math. J. 25 (1958), 265-282.

UNIVERSITY OF MiAMI 